\title{
Assessment of Body Composition in Patients with Multiple Sclerosis: Institutional-Based Cross-Sectional Study \\ Nearmeen M. Rashad ${ }^{1}$, Waleed M. Reda Ashour ${ }^{2}$, Azza H. Abd El-Fatah ${ }^{1}$ \\ Departments ${ }^{1}$ Internal Medicine and ${ }^{2}$ Neurology, Faculty of Medicine, Zagazig University, Sharkia, Egypt \\ * Corresponding author: Nearmeen M. Rashad, Mobile: (+20)1224248642
}

\begin{abstract}
Background: Multiple sclerosis (MS) is the most common progressive neurologic disease all over the world. MS is an immune-mediated disease and there is contradictory data regarding cardiovascular morbidity and mortality risk factors in MS patients.

Objective: To assess the body composition in MS patients, and to explore the associations of anthropometric indices and metabolic risk factors with the phenotypic features of MS.

Patients and Methods: a cross-sectional study included 150 patients fulfilling the diagnostic criteria for MS according to the revised McDonald's criteria 2010, and 145 age and sex-matched healthy controls. Fat mass index (FMI) and free fat mass index (FFMI) were assessed by dual-energy X-Ray absorptiometry (DEXA).

Results: This study was conducted on MS patients with phenotypes; RRMS (82\%) and SPMS (18\%). our results revealed that the distribution of body composition among MS patients was $30 \%$, overweight was $48 \%$ and lean was $22 \%$. Also, MS patients had significantly higher values of systolic and diastolic blood pressure as well as dyslipidemia, obesity indices, and hs-CRP compared to the control group, P-value $<0.001$. Interestingly, there were significantly higher values of the expanded disability status scale (EDSS), dyslipidemia, obesity indices, and hypertension in the obese group compared to other groups.
\end{abstract}

Conclusions: MS patients had higher values of dyslipidemia and obesity indices than control groups. Among MS, the prevalence of overweight patients was higher than lean and obese MS patients.

Keywords: Multiple sclerosis, Body composition, EDSS, FMI

\section{INTRODUCTION}

Multiple sclerosis (MS) is a chronic central nervous system disease with inflammatory and neurodegenerative components. The course of MS is variable and most MS patients initially present with a relapsing-remitting course defined by the acute onset of focal neurologic deficits and focal inflammatory changes. The immunopathological changes include inflammatory demyelinating lesions in the optic nerves, brain, and spinal cord (1), in addition to neurodegeneration lesions ${ }^{(2)}$. According to the patterns of cognitive or physical impairment progression: relapsing-remitting MS (RRMS), primary-progressive MS (PPMS), secondary-progressive MS (SPMS), or progressive-relapsing MS (PRMS) ${ }^{(3)}$.

The prevalence of obesity is rapidly increasing worldwide, According to The World Health Organization (WHO) about 500 million adults are obese, and 1.5 billion adults are overweight, the majority of which in developing countries ${ }^{(4)}$. Obesity also has been raised as a risk factor for MS. ${ }^{(5)}$. Growing evidence highlights the link of systemicobesity inflammatory changes with the MS disease process, particularly in obesity during childhood ${ }^{(6)}$. Interesting studies reported that obesity was also associated with higher levels of C-reactive protein, interleukin-6, and leptin levels ${ }^{(7,8)}$, thus they concluded that a proinflammatory state may affect MS pathogenesis. Interestingly, serum levels of several adipokines, including leptin, adiponectin, and resistin ${ }^{(9)}$, and gut microbiota. are associated with MS ${ }^{(\mathbf{1 0}, \mathbf{1 1})}$.
Obesity contributes significantly to the development and progression of MS which is believed to be mediated by vitamin D deficiency, inflammation, and metabolic syndrome (12). In MS and associated disabilities, despite remarkable advances in the insight into the responsible mechanisms, the etiology remains unknown, the current explorative study was designed to assess the body composition in MS patients, and to explore the associations of anthropometric indices and metabolic risk factors with the phenotypic features of MS.

\section{PATIENTS AND METHODS}

One hundred apparent healthy control subjects and 150 consecutive patients fulfilling the diagnostic criteria for MS according to the revised McDonald's criteria $2010^{{ }^{(13)}}$ were consecutively enrolled to participate in a cross-sectional study. Patients were recruited from outpatient clinics of the Neurology and Internal Medicine Departments of Zagazig University. Both cases and controls were matched for age, gender, and BMI. The MS patients either with RRMS or SPMS. Expanded Disability Status Scale (EDSS) ${ }^{(\mathbf{1 4})}$ of these patients is $<5$. Patients with RRMS were being in the remission period (at least 2 months after the last relapse). The enrolled MS were divided according to body mass index (BMI) to lean, overweight and obese subgroups.

All subjects were subjected to thorough history taking, complete general including an anthropometric and neurological examination. 
Anthropometric variables including body mass index (BMI) calculated as weight in $\mathrm{kg} /$ height in (meters) ${ }^{2}$ and waist circumference $(\mathrm{cm}) /$ hip circumference $(\mathrm{cm})$ (WHR) were measured. Fat mass index (FMI) and free fat mass index (FFMI) were assessed by dual-energy $\mathrm{X}$-Ray absorptiometry (DEXA). Exclusion criteria included pregnancy, lactation, unwillingness to practice acceptable birth control, major depression or suicidal attempt in medical history, and clinically significant heart, liver, renal, or other endocrine diseases.

\section{Ethical approval:}

The study was approved by the Ethics Board of Zagazig University University.Informed consent was obtained from all participants included in the study.

\section{Blood sampling:}

Blood samples were drawn from all subjects after an overnight fast. Sera were separated after $1 \mathrm{~h}$ longstanding and stored at $-80^{\circ} \mathrm{C}$. we measured fasting plasma glucose (FPG) and the lipid profile; total cholesterol (TC), triglycerides (TG), HDL-c and LDLc were measured using Cobas 8000-c702" (Roche Diagnostics GmbH, Mannheim, Germany). HbA1c was measured by Cobas 6000 (Roche Diagnostics $\mathrm{GmbH}$, Mannheim, Germany). C- reactive protein (CRP) was measured using immunoturbidimetric assay on Roche/Hitachi Cobas system (c501) autoanalyzer (Roche Diagnostics, Mannheim, Germany).

\section{Statistical Analysis}

Statistical analyses were performed using the Statistical Package for the Social Sciences for Windows (version 21.0; SPSS Inc., Chicago, IL, USA). Data were expressed using descriptive statistics (mean \pm standard deviation) and were analyzed... Baseline demographic and clinical variables were compared across conditions using one-way analyses of variance (ANOVAs) for continuous variables and chisquare tests of independence for categorical variables. We considered $\mathrm{P}$ to be significant at $<0.05$ with a $95 \%$ confidence interval $(\mathrm{CI})$.

\section{RESULTS}

This cross-sectional study was conducted on 150 consecutive patients of MS (136 females and 14 males) and 145 healthy controls (130 females and 15 males). Among studied MS patients, 123 (82\%) patients had RRMS and 27 (18\%) of the patients had SPMS.

6. Anthropometric and biochemical characteristics of the studied groups.

Obese patients had statistically significantly higher values of SBP, DBP, BMI, waist/hip ratio, FMI, FFMI, TC, TG, LDL, and hs-CRP compared to the control group, (Table 1, $\mathrm{P}<0.01$ ).

Table (1): Anthropometric and biochemical characteristics of the studied groups.

\begin{tabular}{|l|c|c|c|}
\hline \multicolumn{1}{|c|}{ Variables } & $\begin{array}{c}\text { Control group } \\
(\boldsymbol{n = 4 5}\end{array}$ & $\begin{array}{c}\text { MS patients } \\
(\boldsymbol{n}=\mathbf{1 5 0})\end{array}$ & $\boldsymbol{P}$-value \\
\hline Age (years) & $43.48 \pm 8.5$ & $43.25 \pm 7.93$ & 0.9015 \\
\hline Disease duration /year & -- & $6.45 \pm 0.91$ & -- \\
\hline $\begin{array}{l}\text { Phenotypes of MS patients } \\
\text { RRMS }\end{array}$ & -- & $123(82 \%)$ & - \\
SPMS & & $27(18 \%)$ & \\
\hline SBP $(\mathrm{mmHg})$ & $115.54 \pm 6.48$ & $142.23 \pm 8.84$ & $<0.001^{*}$ \\
\hline DBP $(\mathrm{mmHg})$ & $75.6 \pm 2.5$ & $85.75 \pm 8.56$ & $<0.001^{*}$ \\
\hline BMI $(\mathrm{kg} / \mathrm{m} 2)$ & $22.2 \pm 2.014$ & $29.41 \pm 4.21$ & $<0.001^{*}$ \\
\hline Waist circumference $(\mathrm{cm})$ & $0.723 \pm 0.17$ & $1.05 \pm 2.2$ & $<0.001^{*}$ \\
\hline Waist/hip ratio & $0.79 \pm 0.076$ & $1.03 \pm 0.11$ & $<0.001^{*}$ \\
\hline FMI $(\mathrm{kg} / \mathrm{m} 2)$ & $3.34 \pm 0.20$ & $7.57 \pm 2.5$ & $<0.001^{*}$ \\
\hline FFM I $(\mathrm{kg} / \mathrm{m} 2)$ & $18.89 \pm 1.61$ & $21.95 \pm 3.87$ & $<0.001^{*}$ \\
\hline TC $(\mathrm{mg} / \mathrm{dl})$ & $185.06 \pm 19.87$ & $209.3 \pm 8.87$ & $<0.001^{*}$ \\
\hline TG $(\mathrm{mg} / \mathrm{dl})$ & $133.62 \pm 16.75$ & $177.54 \pm 15.0$ & $<0.001^{*}$ \\
\hline LDL .c $(\mathrm{mg} / \mathrm{dl})$ & $102.22 \pm 6.79$ & $121.48 \pm 3.7$ & $<0.001^{*}$ \\
\hline HDL .c $(\mathrm{mg} / \mathrm{dl})$ & $47.82 \pm 4.26$ & $37.96 \pm 8.38$ & $<0.001^{*}$ \\
\hline FPG $(\mathrm{mg} / \mathrm{dl})$ & $84.62 \pm 6.276$ & $84.93 \pm 10.03$ & 0.379 \\
\hline hs-CRP $(\mu \mathrm{g} / \mathrm{ml})$ & $0.96 \pm 0.06$ & $6.14 \pm 1.89$ & $<0.001^{*}$ \\
\hline
\end{tabular}

MS; Multiple sclerosis, RRMS; relapsing-remitting multiple sclerosis, SPMS; secondary-progressive multiple sclerosis, EDSS; Expanded Disability Status Scale, SBP; systolic blood pressure; DBP; diastolic blood pressure, FMI; fat mass index, FFMI; fatfree mass index-c; high-density lipoprotein cholesterol, LDL-c; low-density lipoprotein cholesterol, FPG; fasting plasma glucose, ${ }^{*} P<0.001$ when compared MS patients with the control group. 


\section{The prevalence of body composition among patients with MS}

Among the MS group, 33(22\%) patients were lean, 72(48\%) patients were overweight, and 45 (30\%) patients were obese figure 1 .

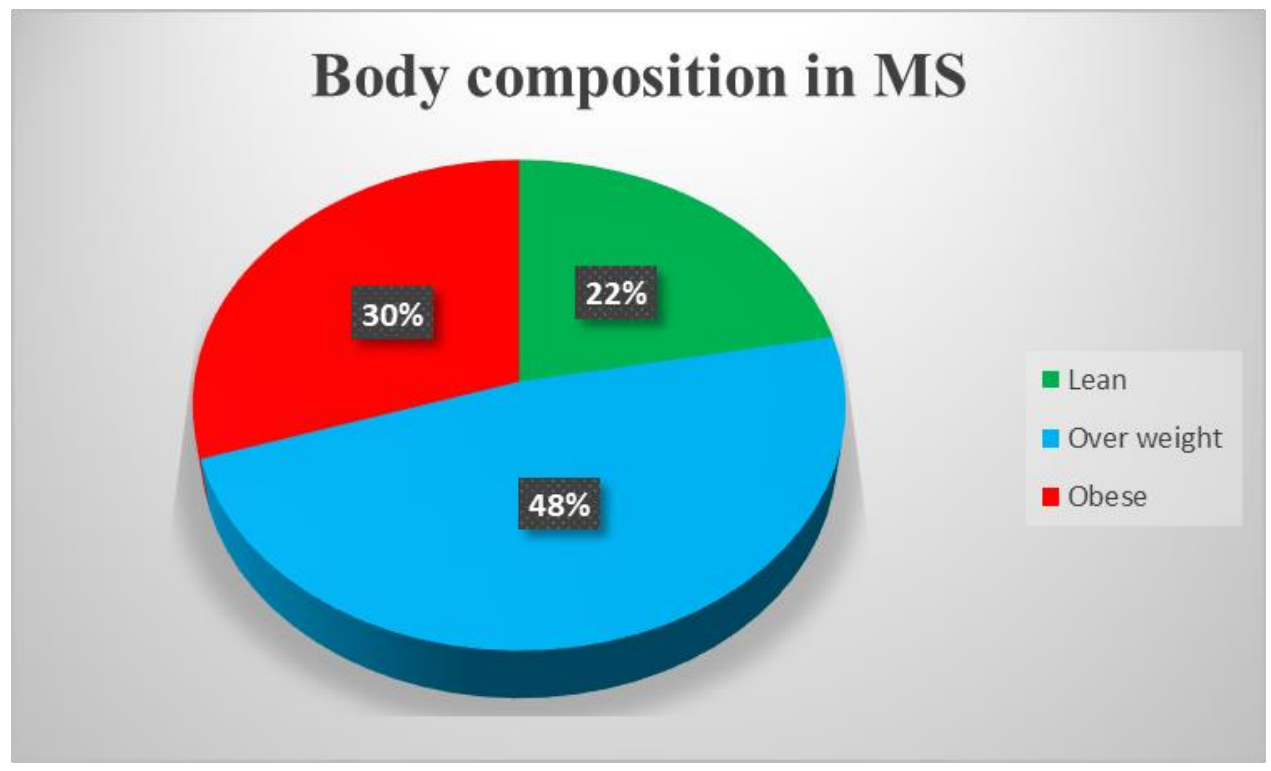

Figure (1): The prevalence of body composition among patients with MS

\section{Characteristics of MS patients stratified according to BMI}

Obese patients had statistically significant higher values of age, duration of disease, SBP, DBP, BMI, waist/hip ratio, FMI, FFMI, TC, TG, LDL, and hs-CRP compared to lean and overweight MS patients, (Table 2, P< $0.05)$.

Table (2): Characteristics of MS patients stratified according to BMI

\begin{tabular}{|c|c|c|c|c|c|c|}
\hline \multirow[t]{2}{*}{ Variable } & \multicolumn{3}{|c|}{$\begin{array}{c}\text { MS } \\
(\mathbf{N}=150)\end{array}$} & \multirow[t]{2}{*}{ P 1} & \multirow[t]{2}{*}{$\mathbf{P 2}$} & \multirow[t]{2}{*}{ P3 } \\
\hline & $\begin{array}{c}\text { Lean } \\
(n=33)\end{array}$ & $\begin{array}{c}\text { Overweight } \\
(\mathrm{n}=72)\end{array}$ & $\begin{array}{l}\text { Obese } \\
(n=45)\end{array}$ & & & \\
\hline Age(years) & $43.28 \pm 8.5$ & $38.8 \pm 7.93$ & $50.25 \pm 9.9$ & 0.014 & 0.001 & $<0.001$ \\
\hline Duration of MS & $7.0 \pm 1.57$ & $9.10 \pm 0.51$ & $13.41 \pm \quad 1.5$ & $<0.001^{*}$ & $<0.001^{*}$ & .071 \\
\hline EDSS & $0.56 \pm 0.01$ & $2.29 \pm 0.31$ & $4.05 \pm 0.456$ & $<0.001^{*}$ & $<0.001^{*}$ & $<0.001^{\prime}$ \\
\hline SBP $(\mathrm{mmHg})$ & $117.8 \pm \quad 19.7$ & $145.6 \pm \quad 21.9$ & $152.2 \pm 18.9$ & $<0.001^{*}$ & $<0.001^{*}$ & $<0.001^{\prime}$ \\
\hline $\mathrm{DBP}(\mathrm{mmHg})$ & $75.0 \pm 7.57$ & $84.10 \pm \quad 10.51$ & $95.41 \pm \quad 13.5$ & $<0.001^{*}$ & $<0.001^{*}$ & $<0.001^{\prime}$ \\
\hline BMI (kg/m2) & $22.1 \pm 1.26$ & $28.13 \pm 1.2$ & $36.55 \pm 4.3$ & $<0.001^{*}$ & $<0.001^{*}$ & $<0.001^{*}$ \\
\hline Waist/hip ratio & $0.99 \pm 0.096$ & $1.03 \pm 0.087$ & $1.13 \pm 0.10$ & $<0.001^{*}$ & $<0.001^{*}$ & $<0.001^{*}$ \\
\hline FMI $\left(\mathrm{kg} / \mathrm{m}^{2}\right)$ & $4.9 \pm 0.52$ & $6.7 \pm 0.4$ & $11.1 \pm 1.28$ & $<0.001^{*}$ & $<0.001^{*}$ & $<0.001^{\prime}$ \\
\hline FFMI $\left(\mathrm{kg} / \mathrm{m}^{2}\right)$ & $\begin{array}{ll}17.2 \pm 9 & .53 \\
\end{array}$ & $21.6 \pm 0.79$ & $25.5 \pm 1.02$ & $<0.001^{*}$ & $<0.001^{*}$ & $<0.001^{\prime}$ \\
\hline TC (mg/dl) & $185.4 \pm$ & $215.5 \pm \quad 2.9$ & $217.2 \pm \quad 3.1$ & $<0.001^{*}$ & $<0.001^{*}$ & 0.754 \\
\hline TG (mg/dl) & $132.2 \pm \quad 4.8$ & $169.5 \pm \quad 5.1$ & $221.1 \pm \quad 5.2$ & $<0.001^{*}$ & $<0.001^{*}$ & $<0.001$ \\
\hline LDL .c (mg/dl) & $102.0 \pm 14.5$ & $132.4 \pm \quad 2.7$ & $113.1 \pm \quad 2.9$ & $<0.001^{*}$ & 0.05 & $<0.001$ \\
\hline HDL .c (mg/dl) & $48.6 \pm 5 \quad .51$ & $7.7 \pm 5.52$ & $31.8 \pm 0.72$ & $<0.001^{*}$ & $<0.001^{*}$ & $<0.001^{\prime}$ \\
\hline FPG (mg/dl) & $85.3 \pm 0 \quad 8.6$ & $85.68 \pm \quad 7.2$ & $87.1 \pm 8.5^{\#}$ & 0.710 & 0.733 & 0.412 \\
\hline hs-CRP $(\mu \mathrm{g} / \mathrm{ml})$ & $3.9 \pm 0.52$ & $5.7 \pm 1.4$ & $7.1 \pm 1.78$ & $<0.001^{*}$ & $<0.001^{*}$ & $<0.001$ \\
\hline
\end{tabular}

MS; Multiple sclerosis, EDSS; Expanded Disability Status Scale; SBP: systolic blood pressure; DBP: diastolic blood pressure; FMI, fat mass index; FFMI, fat-free mass index; HDL-c: high-density lipoprotein cholesterol; LDL-c: low-density lipoprotein cholesterol; FPG fasting plasma glucose. ${ }^{1} \mathrm{P}<0.001$ when compared group overweight with lean group, ${ }^{2} \mathrm{P}<0.001$ when compared obese with lean, ${ }^{3} \mathrm{P}<0.001$ when compared obese with overweight. 


\section{Comparison of EDSS among MS patients}

Among MS patients, obese MS patients had significantly higher values of EDSS expression levels (4.05 \pm 0.456$)$ compared to overweight $(2.29 \pm 0.31)$ and lean subgroups $(0.56 \pm 0.01)$ (figure 2$), \mathrm{p}<0.001^{*}$.

\section{EDSS}

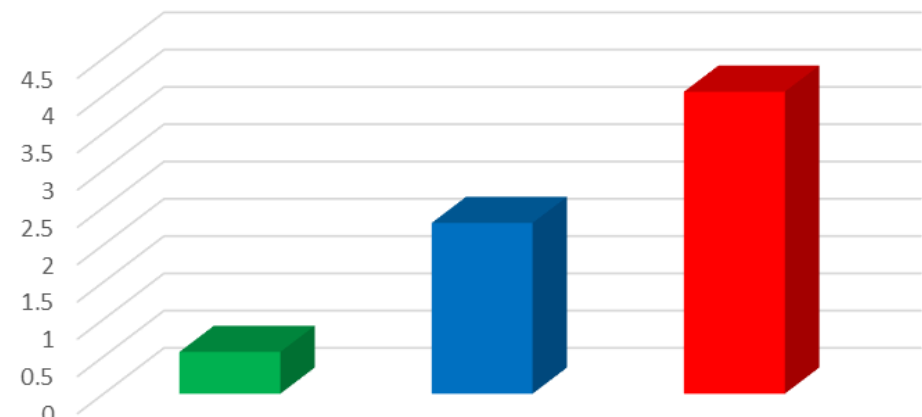

Lean Over weight Obese

Figure (2): Comparison of EDSS among MS patients

Correlation between EDSS and clinical, anthropometric indices as well as other metabolic studied parameters of MS patients.

Among MS groups, $(n=150)$, EDSS values were significantly positively correlated with SBP, DBP, TC, TG, LDL, as well as anthropometric measures; BMI, FMI, FFMI, and waist/circumference. Furthermore, EDSS values were significantly negatively correlated with HDL, P-value $<0.01 *$ (Table 3 ).

Table (3): Pearson correlation coefficient between circulatory EDSS values with clinical, anthropometric indices as well as other metabolic studied parameters of among MS patients.

\begin{tabular}{|l|l|c|}
\hline \multirow{2}{*}{ BMI } & & EDSS values \\
\hline \multirow{2}{*}{ FMI $\left(\mathrm{kg} / \mathrm{m}^{2}\right)$} & $r$ & 0.761 \\
\cline { 2 - 3 } & $P$ & $<0.001^{*}$ \\
\hline \multirow{2}{*}{ FFMI $\left(\mathrm{kg} / \mathrm{m}^{2}\right)$} & $r$ & 0.868 \\
\cline { 2 - 3 } & $P$ & $<0.001^{*}$ \\
\hline \multirow{2}{*}{ waist/hip ratio } & $r$ & 0.642 \\
\hline \multirow{2}{*}{ SBP $(\mathrm{mmHg})$} & $r$ & $<0.001^{*}$ \\
\cline { 2 - 3 } & $P$ & 0.745 \\
\hline \multirow{2}{*}{ DBP (mmHg) } & $r$ & $<0.001^{*}$ \\
\cline { 2 - 3 } & $P$ & 0.483 \\
\hline \multirow{2}{*}{ TG (mg/dl) } & $r$ & $<0.001^{*}$ \\
\cline { 2 - 3 } & $P$ & 0.482 \\
\hline \multirow{2}{*}{ HDL .c (mg/dl) } & $r$ & $<0.001^{*}$ \\
\cline { 2 - 3 } & $P$ & 0.387 \\
\hline \multirow{2}{*}{ TC $(\mathrm{mg} / \mathrm{dl})$} & $r$ & $<0.001^{*}$ \\
\cline { 2 - 3 } & $P$ & 0.606 \\
\hline \multirow{2}{*}{ LDL (mg/dl) } & $r$ & $<0.001^{*}$ \\
\cline { 2 - 3 } & $P$ & 0.387 \\
\hline FPG (mg/dl) & $r$ & $<0.001^{*}$ \\
\cline { 2 - 3 } & $P$ & 0.261 \\
\cline { 2 - 3 } & $r$ & $<0.001^{*}$ \\
\hline \multirow{2}{*}{} & $P$ & 0.012 \\
\hline
\end{tabular}


Linear regression analysis among MS to test the main independent variables against EDSS values.

Linear regression analysis revealed that HDL, BMI, and duration of MS were the main predictors of EDSS values among other parameters, P-value $<0.001^{*}$.

Table (4): Linear regression analyses to test the influence of the main independent variables against EDSS values (dependent variable) in MS patients

\begin{tabular}{|c|c|c|c|c|c|c|c|}
\hline \multirow[t]{2}{*}{ Model } & \multirow{2}{*}{\begin{tabular}{|c}
$\begin{array}{c}\text { Unstandardized } \\
\text { Coefficients }\end{array}$ \\
B
\end{tabular}} & \multirow[b]{2}{*}{ S.E } & \multirow{2}{*}{$\begin{array}{c}\begin{array}{c}\text { Standardized } \\
\text { Coefficients }\end{array} \\
\text { Beta }\end{array}$} & \multirow[t]{2}{*}{$\mathbf{t}$} & \multirow[t]{2}{*}{ P-value } & \multicolumn{2}{|c|}{ 95\% C.I. } \\
\hline & & & & & & $\begin{array}{l}\text { Lower } \\
\text { Bound }\end{array}$ & $\begin{array}{l}\text { Upper } \\
\text { Bound }\end{array}$ \\
\hline (Constant) & 27.245 & 3.782 & & 7.203 & $<0.001 *$ & 19.769 & 34.721 \\
\hline HDL & 0.072 & 0.022 & 0.089 & 3.215 & $<0.001 *$ & 0.028 & 0.116 \\
\hline FPG & 0.028 & 0.034 & .015 & .826 & 0.410 & 0.039 & 0.096 \\
\hline BMI & 3.853 & 0.127 & 3.380 & 30.350 & $0.001 *$ & 3.602 & 4.104 \\
\hline Duration of MS & 8.703 & 00.341 & -3.323 & -25.49 & $0.001 *$ & -9.378 & -8.028 \\
\hline
\end{tabular}

$* \mathrm{P}<0.05$

\section{DISCUSSION}

A preponderance of evidence suggests that MS results from a combination of genetic predisposition and environmental influences ${ }^{(15)}$. Intriguing reports are investigating the risk factors of MS include; low vitamin D levels ${ }^{(\mathbf{1 6})}$, sun exposure ${ }^{(\mathbf{1 7})}$, smoking ${ }^{(\mathbf{1 8})}$, and viral exposure ${ }^{(\mathbf{1 9})}$.

A growing body of evidence has corroborated that the pathophysiology of MS is complex and incompletely understood. Obesity is a growing health concern that has become an epidemic all over the world. There is a bi-directional relationship between obesity and MS. Substantial evidence implicate inflammation as a critical mediator in the pathophysiology of MS. Gene expression patterns in different clinical phenotypes may increase our understanding of disease processes Identifying a successful biomarker depends inevitably on fully understanding the pathophysiology underlying the disease. Thus, we aimed in the current study to assess the body composition in MS patients, and to explore the associations of anthropometric indices and metabolic risk factors with the phenotypic features of MS.

This study was conducted on MS patients with phenotypes; RRMS (82\%) and SPMS (18\%). The interesting result of our study was that the prevalence of obesity among MS patients was $30 \%$, overweight was $48 \%$ and lean was $22 \%$. The metabolic characteristics of this study population were varied in the different MS phenotypes through the progression from lean to overweight and obese, subjects had worse metabolic profiles, which were consistent with increased obesity indices and insulin resistance.

According to Khurana et al. ${ }^{(20)}$ findings, about $50 \%$ of patients with MS are overweight or obese. Interestingly, a study conducted by Gianfrancesco et al. ${ }^{(21)}$ suggested that childhood and adolescence obesity increase the risk of MS in females after accounting for established genetic and environmental risk factors.

An interesting Egyptian study was conducted to evaluate the association between obesity, vitamin, and MS. they observed that vitamin D deficiency and overweight are predominant among Egyptian MS patients (22). Another study on risk factors of MS detected a significant interaction between adolescent $\mathrm{BMI}$ and infectious mononucleosis associated with MS ${ }^{\text {(23) }}$. Huppke et al. ${ }^{(24)}$ demonstrated that the increase in pediatric MS risk appeared to be associated with obesity, and obese patients did not respond well to first-line medications; altered pharmacokinetics appeared to be most likely factors in treatment response, and they recommended that achieving a healthy weight or adjusting the dose according to BMI could improve therapy response. A similar study conducted on adolescence and young adulthood found obesity was associated with an earlier age at multiple sclerosis onset ${ }^{(25)}$.

The current research was designed to evaluate the cardiometabolic risk factors among MS patients compared to controls and we detected that MS patients had significantly higher values of SBP, DBP, BMI, waist/hip ratio, FMI, FFMI, TC, TG, LDL, and hs-CRP compared to the control group. On the other hand, HDL levels were significantly lower values in MS patients compared to controls.

Similar to our study, Hussein et al reported a higher prevalence of hyperglycemia compared to the general population ${ }^{(26)}$. Though Marrie et al. ${ }^{(27)}$ found a similar prevalence of hyperglycemia in MS patients compared to the general population. Even more interestingly, patients with MS and type 2 DM may have a worse progression of disability compared to people without DM ${ }^{(28)}$.

A study conducted by Almdal et al. (29) observed that hypertension leads to faster progression of MS and clinical disability. Regarding dyslipidemia and MS, there is contradictory data Weinstock- 
Guttman et al revealed dyslipidemia is associated with MS disability and MRI outcomes ${ }^{(30)}$ as LDL has a negative impact on acute inflammatory activity and disease course in MS patients, furthermore, EDSS worsening was associated with dyslipidemia ${ }^{(31)}$, on the other hand, higher levels of HDL have a positive effect on the course of MS, which is consistent with the antioxidant and anti-inflammatory properties of HDL (32).

A population-based controlled study was designed to evaluate the comorbidities amongst patients with MS and they found that the risk of ischemic heart disease, stroke, or heart failure is significantly increased in MS patients (33, 34). Interestingly, MS is associated with an almost threefold increase in the risk for death, according to Lalmohamed et al ${ }^{(35)}$ results suggesting that this is due to cardiovascular disease.

We in this study attempted to pierce out the impact of obesity on cardiometabolic, MS-related disabilities and inflammatory markers among MS patients and we observed that obese MS patients had statistically significant higher values of EDSS, duration of disease, SBP, DBP, BMI, waist/hip ratio, FMI, FFMI, TC, TG, and LDL compared to lean and overweight MS patients

Similar to our study, Zamzam et al. (22) revealed that the EDSS was positively correlated to the BMI and negatively correlated to $25(\mathrm{OH}) \mathrm{D}$ levels in Egyptian patients with MS.

\section{CONCLUSION}

MS patients had higher values of dyslipidemia and obesity indices than control groups. the prevalence of overweight patients with MS was higher than lean and obese MS patients.

Competing interests: The authors declare that they have no competing interests to disclose.

Acknowledgment: The authors would like to thank all the patients and healthy controls accepted to participate in this study. The authors would like to thank colleagues in the Radiology Department, Zagazig University Hospitals for their help in the current study. Funding: This research did not receive any specific grant from funding agencies in the public, commercial, or not-for-profit sectors.

\section{REFERENCES}

1. Katz S (2015): Classification, diagnosis, and differential diagnosis of multiple sclerosis. Curr Opin Neurol., 28(3):193-205

2. Calabrese M, Atzori M, Bernardi V et al. (2007): Cortical atrophy is relevant in multiple sclerosis at clinical onset. J Neurol., 254:1212-16.

3. Fisniku L, Chard D, Jackson J et al. (2008): Gray matter atrophy is related to long-term disability in multiple sclerosis. Ann Neurol., 64(3):247-54.
4. World Health Organization (2017): Obesity and Overweight. Available online: http://www.who.int/mediacentre/factsheets/fs311/en/w ww.who.int/topics/obesity/en

5. Gianfrancesco $M$, Glymour $M$, Walter $S$ et al. (2017): Causal effect of genetic variants associated with body mass index on multiple sclerosis susceptibility. Am J Epidemiol., 185 (3): 162-171.

6. Barbati A, Osculati F, Silvagni D et al. (2006): Obesity and inflammation: evidence for an elementary lesion. Pediatrics, 117(1):220-3.

7. Kanneganti T, Dixit V (2012): Immunological complications of obesity. Nat Immunol., 13(8):707-12.

8. Versini M, Jeandel P, Rosenthal E et al. (2014): Obesity in autoimmune diseases: not a passive bystander. Autoimmun Rev., 13(9):981-1000.

9. Kau A, Ahern P, Griffin N et al. (2011): Human nutrition, the gut microbiome, and the immune system. Nature, 474(7351):327-36.

10. Lee $Y$, Menezes J, Umesaki $Y$ et al. (2011): Proinflammatory T-cell responses to gut microbiota promote experimental autoimmune encephalomyelitis. Proc Natl Acad Sci USA., 108(1):4615-22.

11. Tsai F, Coyle W (2009): The microbiome and obesity: is obesity linked to our gut flora? Curr Gastroenterol Rep., 11(4):307-13.

12. Hayes E, Ntambi J (2020): Multiple Sclerosis: Lipids, Lymphocytes, and Vitamin D. Immunometabolism, 2(3): 200019.

13. Polman C, Reingold S, Banwell B et al. (2011): Diagnostic criteria for multiple sclerosis: 2010 revisions to the McDonald criteria. Ann Neurol., 69(2):292-302.

14. Kurtzke J (1983): Rating neurologic impairment in multiple sclerosis: an expanded disability status scale (EDSS). Neurology, 33(11):1444-52.

15. Hedström A (2016): Environmental factors and their interactions with risk genotypes in MS susceptibility. Curr Opin Neurol., 29(3):293-8.

16. Munger K, Levin L, Hollis B et al. (2006): Serum 25hydroxyvitamin D levels and risk of multiple sclerosis. JAMA., 296(23):2832-8.

17. Van der Mei I, Ponsonby A, Dwyer T et al. (2003): Past exposure to sun, skin phenotype, and risk of multiple sclerosis: a case-control study. BMJ., 327(7410):316.

18. Hedström A, Olsson T, Alfredsson L (2016): Smoking is a major preventable risk factor for multiple sclerosis. Mult Scler Mult Scler., 22(8):1021-6.

19. Munger K, Bentzen J, Laursen B et al. (2013): Childhood body mass index and multiple sclerosis risk: a long-term cohort study. Mult Scler., 19(10):1323-9.

20. Khurana S, DiGiacomo A, Rohini $\mathrm{V}$ et al. (2006): Prevalence of overweight and obesity among Veterans with multiple sclerosis. Int J MS Care, 8:59-63.

21. Gianfrancesco M, Acuna B, Shen L et al. (2014): Obesity during childhood and adolescence increases susceptibility to multiple sclerosis after accounting for established genetic and environmental risk factors. Obesity Research and Clinical Practice, 8:435-447.

22. Zamzam D, Foad M, Swelam M et al. (2019): Vitamin $\mathrm{D}$ and body mass index in Egyptian multiple sclerosis patients. Mult Scler Relat Disord., 28:313-316. 
23. Hedström A, Lima Bomfim I, Hillert J et al. (2015): Obesity interacts with infectious mononucleosis in the risk of multiple sclerosis. Eur J Neurol., 22(3):578-38.

24. Huppke B, Ellenberger D, Hummel H et al. (2019): Association of Obesity with Multiple Sclerosis Risk and Response to First-line Disease-Modifying Drugs in Children. JAMA Neurol., 76(10):1157-1165.

25. Kavak K, Teter B, Hagemeier J et al. (2015): New York State Multiple Sclerosis Consortium. Higher weight in adolescence and young adulthood is associated with an earlier age at multiple sclerosis onset. Mult Scler., 21(7):858-65.

26. Hussein W, Reddy $S$ (2006): Prevalence of diabetes in patients with multiple sclerosis. Diabetes Care, 29:1984-1985.

27. Marrie R, Yu B, Leung S et al. (2012): The rising prevalence of vascular comorbidities in multiple sclerosis: validation of administrative definitions for diabetes, hypertension, and hyperlipidemia. Mult Scler., 18:1310-1319.

28. Wong E, Backholer K, Gearon E et al. (2013): Diabetes and risk of physical disability in adults: a systematic review and meta-analysis. Lancet Diabetes Endocrinol., 1:106-114.

29. Al Modal T, Scharlng H, Jensen J et al. (2004): The independent effect of type 2 diabetes mellitus on ischemic heart disease, stroke, and death: a populationbased study of 13000 men and women with 20 years of follow up. Arch Intern Med., 164:1422-1426.

30. Weinstock-Guttman B, Zivadinov R, Mahfooz $\mathbf{N}$ et al. (2011): Serum lipid profiles are associated with disability and MRI outcomes in multiple sclerosis. J Neuroinflammation, 8:127-132.

31. Palavra F, Marado D, Mascarenhas- Melo $F$ et al. (2013): New markers of early cardiovascular risk in multiple sclerosis patients: oxidized-LDL correlates with clinical staging. Dis Markers, 34:341-348.

32. Tettey P, Simpson S, Taylor B et al. (2014): Vascular comorbidities in the onset and progression of multiple sclerosis. J Neurol Sci., 347:23-33.

33. Kang J, Chen Y, Lin H (2010): Comorbidities amongst patients with multiple sclerosis: A populationbased controlled study. Eur J Neurol., 17:1215-1219.

34. Lalmohamed A, Bazelier M, Van Staa T et al. (2012): Causes of death in patients with multiple sclerosis and matched referent subjects: A population-based cohort study. Eur J Neurol., 19:1007-1014.

35. Jadidi E, Mohammadi M, Moradi T (2013): High risk of cardiovascular diseases after diagnosis of multiple sclerosis. Mult Scler., 19:1336-1340. 